the leak site was sutured at the skin level after $24 \mathrm{~h}$ of persistent leakage. The patient was discharged home the next day completely asymptomatic. She had the suture removed 3 days later, without any recurrence of the leakage. She didn't report any symptoms during a two-month follow-up.

Conclusions This report presents another case of a CSF fistula after a neuraxial block and highlights the success of a conservative approach that avoided not only a blood patch, but also a surgical procedure, in an asymptomatic young woman.

\section{FLUOROSCOPIC APPROACH TO NEURAXIAL ANAESTHESIA IN A PATIENT OF KYPHOSCOLIOSIS}

S Gupta*, R Mane. Jawaharlal Nehru Medical College, KAHER, Belagavi, India

\subsection{6/rapm-2021-ESRA.12}

Background and Aims Kyphoscoliosis is a complex deformity of the spine resulting in lateral curvature and rotation of the vertebrae and a deformity of the rib cage. There is usually secondary involvement of the cardio-respiratory and neurologic systems.

Neuraxial block is routinely performed without the aid of imaging modalities and approached blindly for placement of epidural catheter. Kyphoscoliosis poses a remarkable challenge to administration of neuraxial anaesthesia and may result in multiple pricks and complications such as nerve injury and hematoma formation.

We aimed to assess the feasibility and success of using fluoroscopy guidance for administration of epidural anaesthesia in a case of severe kyphoscoliosis.

Methods A 18-year-old female $132 \mathrm{~cm}$ tall, with severe kyphoscoliosis was posted for Cholecystectomy. She had severe dextrorotatory-thoracic scoliosis [Cobb angle- $62^{\circ}$ ] and a compensatory levorotatory-lumbar curve $\left[45^{\circ}\right]$. Respiratory examination showed restricted breathing with $\mathrm{B} / \mathrm{L}$ wheeze. PFT showed a restrictive pattern. Open cholecystectomy was planned under Epidural Anaesthesia. Serial MRI films were assessed to determine the direction of placement of the epidural needle which was enhanced with the use of fluroscopy guidance during the intraoperative period.

Results Intraoperative period assessed in terms of patient compliance and adequate surgical anaesthesia was satisfactory.

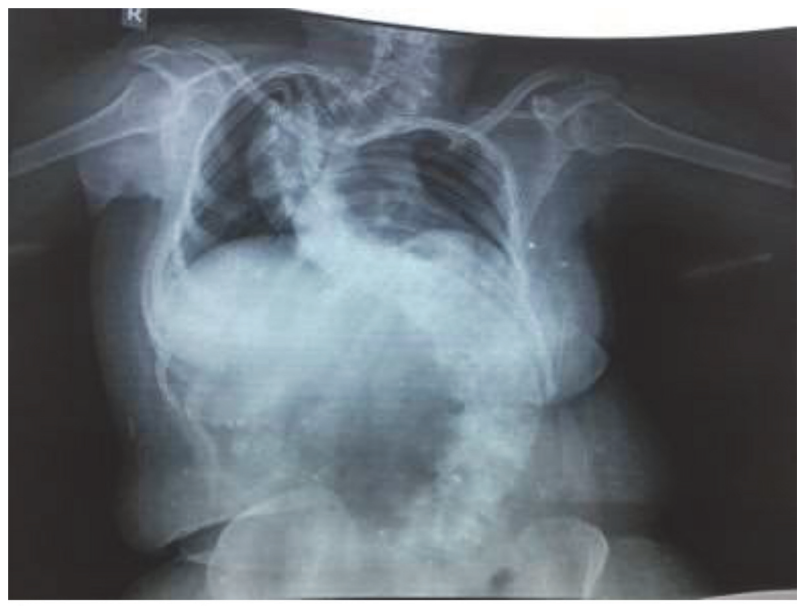

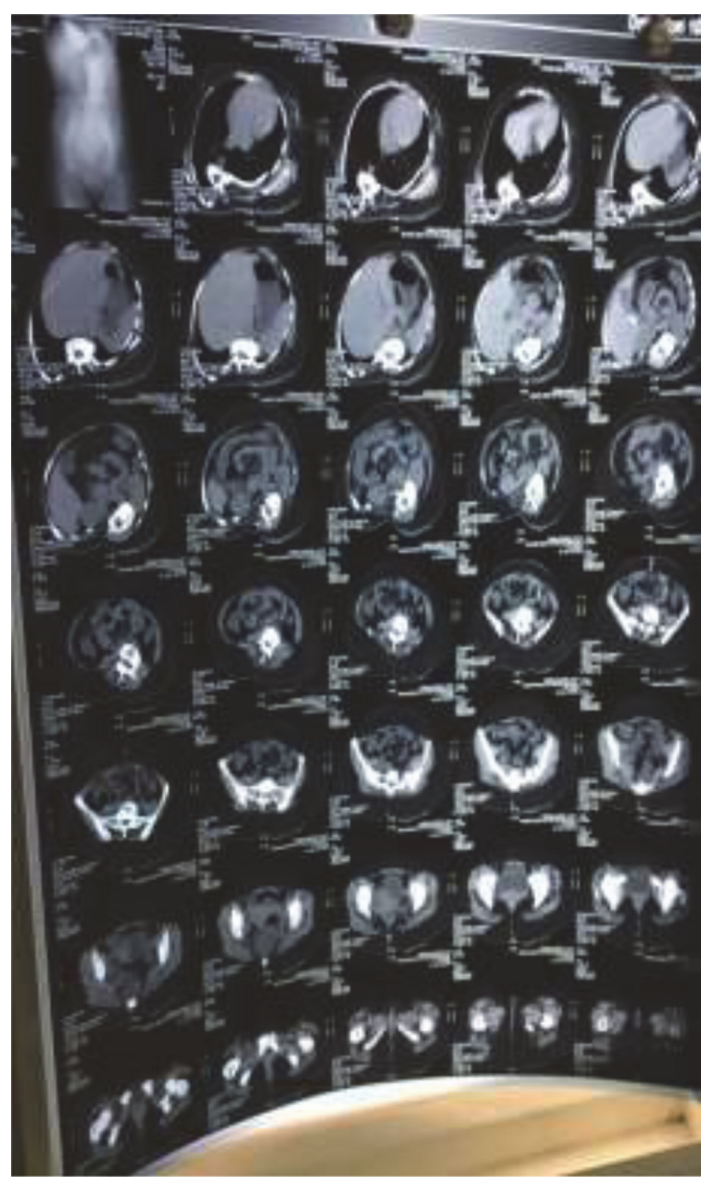

Abstract 12 Figure 2

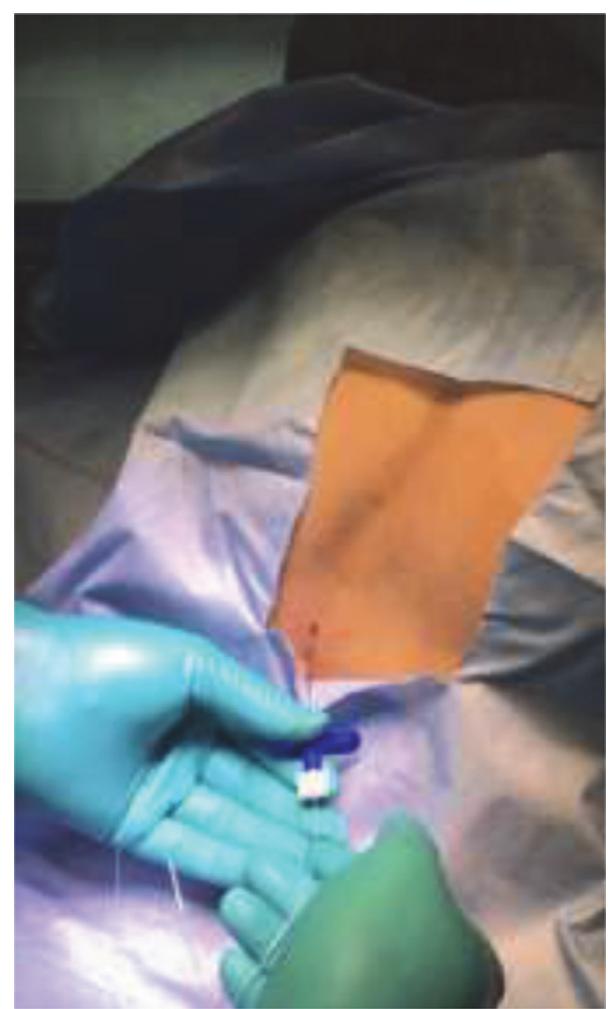

Abstract 12 Figure 3 
Intraoperative use of analgesics was significantly reduced. Epidural analgesia was continued for 2 days postoperatively.

Conclusions Despite various challenges, this case of severe kyphoscoliosis was successfully managed with fluoroscopyguided epidural anaesthesia. Fluoroscopy is a well-established imaging modality and its knowledge adds to the Anaesthesiologist armamentarium and offers an opportunity to provide neuraxial anaesthesia in patients with anticipated difficulty.

\section{CONTINUOUS ERECTOR SPINAE PLANE BLOCK AS MULTIMODAL ANALGESIA AND INTRAOPERATIVE HEMODYNAMIC STABILITY IN OPEN NEPHRECTOMY FOR LIVING DONOR KIDNEY TRANSPLANT}

R Rahmi* ${ }^{*}$ RB Sukmono. Universitas Indonesia, Jakarta, Indonesia

\subsection{6/rapm-2021-ESRA.13}

Background and Aims Open nephrectomy is associated with moderate to severe postoperative pain.1 Erector spinae plane block (ESPB) provides effective analgesia for various surgical procedure but only a few reports on ESPB used for pain control in open nephrectomy.2 We reported two cases of

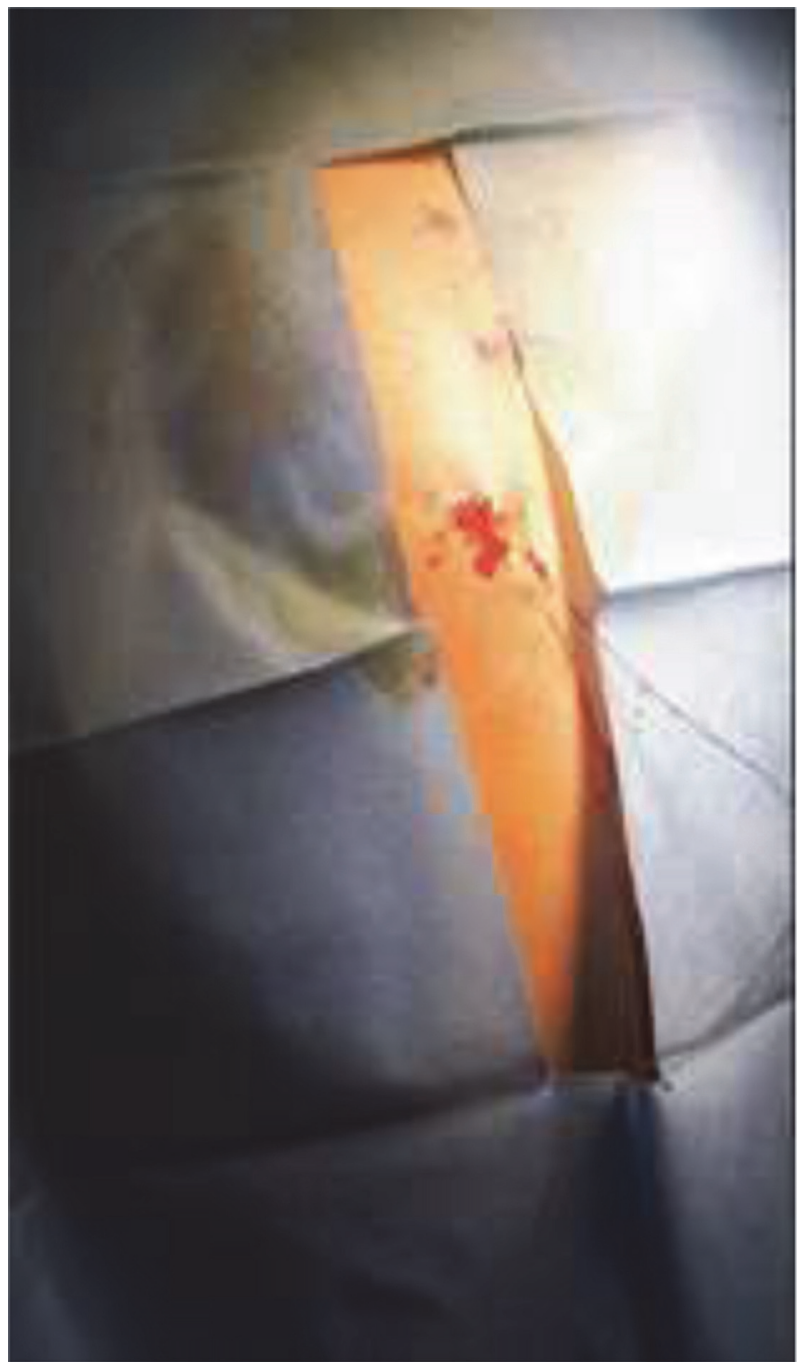

Abstract 13 Figure 1 continuous ESPB as multimodal analgesia and intraoperative hemodynamic stability in open nephrectomy for living donor kidney transplant.

Methods Two adult patients underwent open nephrectomy for living donor renal transplant. ESPB was performed before general anesthesia with ultrasound guided using high frequency linear probe at level of T8-T9. After transversus process and erector spinae muscle were identified, hydro dissection with $2 \mathrm{ml}$ of normal saline was used to confirm the correct needle tip position. 18-gauge epidural catheter then inserted into the plane between erector spinae and transverse process. $20 \mathrm{ml}$ local anesthetic 0,25\% levobupivacaine was injected and spread of local anesthetic under erector spinae muscle was seen by ultrasound. For postoperative pain control, patients were administered $10 \mathrm{ml}$ levobupivacaine $0,125 \%$ via catheter at the end of surgery, continuous levobupivacaine $0,125 \% 6$

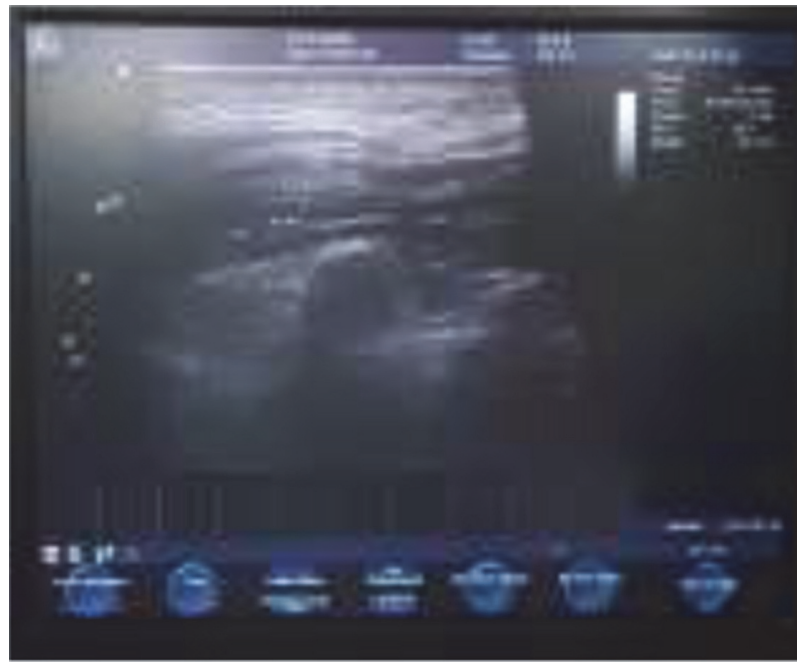

Abstract 13 Figure 2

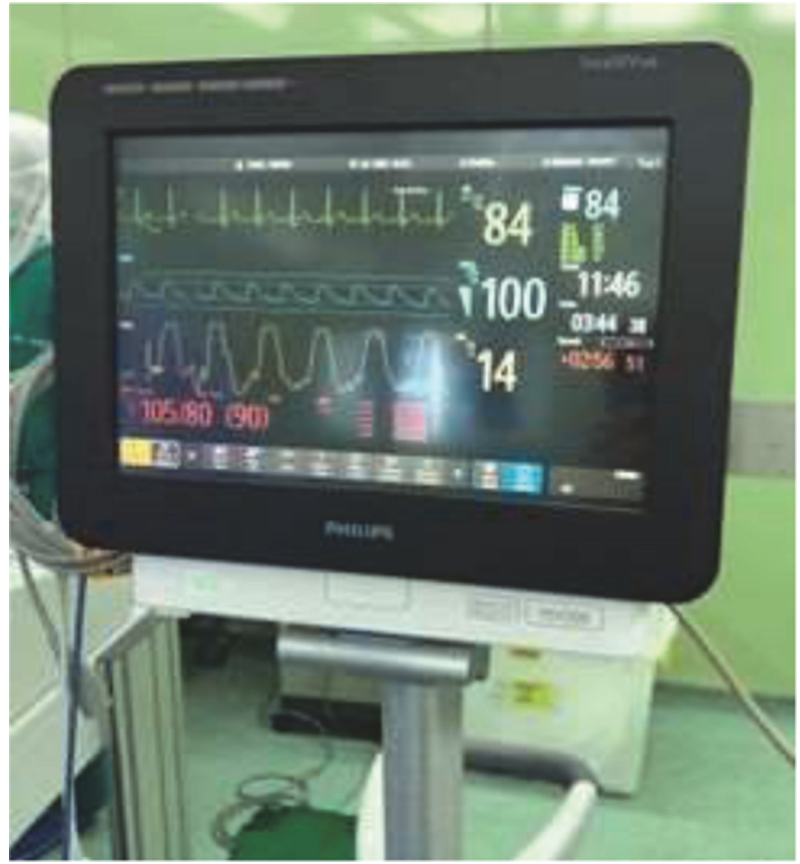

Abstract 13 Figure 3 